PROCEEDINGS OF THE

AMERICAN MATHEMATICAL SOCIETY

Volume 131, Number 3, Pages 781-791

S 0002-9939(02)06592-9

Article electronically published on July 17, 2002

\title{
EFFECTS OF ABSOLUTE CONTINUITY IN FEYNMAN'S OPERATIONAL CALCULUS
}

\author{
LANCE NIELSEN
}

(Communicated by N. Tomczak-Jaegermann)

\begin{abstract}
We investigate the effects of having an absolute continuity relation between the time-ordering measures in Feynman's operational calculus. In particular, we obtain some theorems concerning the formation of functions of several noncommuting operators or operator-valued functions under specific absolute continuity assumptions on the time-ordering measures.
\end{abstract}

\section{INTRODUCTION}

In the setting of Feynman's operational calculus we are concerned with the formation of functions of several noncommuting operators. While this problem is an important one in many areas of mathematics and physics, it is also a difficult one. For suppose that $f(x, y)=x y$. If we wish to evaluate $f(A, B)$ for $A$ and $B$ noncommuting linear operators, there is clearly an ambiguity as to how to proceed. Is the result $A B, B A, \frac{1}{2}(A B+B A)$, or some other quantity? (This ambiguity is present even if the operators involved are self-adjoint and bounded.) This problem was of concern to Richard Feynman [2] due to his interest in quantum theory where the observables that one considers are self-adjoint operators which generally do not commute with one another. To cope with this ambiguity, Feynman invented some 'rules' for the formation of functions of noncommuting operators. These are:

(R1) Attach time indices to operators to indicate the order in which they act in products. (Note: Operators sometimes come with time indices naturally attached.) Feynman's time ordering convention was that an operator with a smaller (or earlier) time index should act before one with a larger (or later) index no matter how they are ordered on the page.

(R2) With time indices attached, form functions of these operators as if they were commuting. (If one stops naively at this point, the 'equality' involved is usually false. For example, it might say that $e^{A+B}=e^{A} e^{B}$ even though $A$ and $B$ do not commute.)

(R3) After (R2) is completed, 'disentangle' the resulting expression; that is, restore the conventional ordering of the operators. In practice, this means to manipulate the expression (if possible) until the ordering on the page coincides with the time ordering.

Received by the editors July 10, 2001 and, in revised form, October 10, 2001.

2000 Mathematics Subject Classification. Primary 47A60, 46H30; Secondary 47A56, 47B38.

Key words and phrases. Functional calculus, disentangling, operational calculus. 
A much more extensive introduction to Feynman's operational calculus including a discussion of its connection with the Feynman path integral can be found in the book of Johnson and Lapidus [7; see especially Chapter 14. Further work which will soon be referenced below can also be consulted.

The papers of Jefferies and Johnson 3 and of Jefferies, Johnson, and Nielsen [5] are the most closely related to this paper and serve to supply the initial definitions below and, in particular, the definition of the disentangling map. (See Definitions 2.3 and 2.4 below.) In the first of these papers, Jefferies and Johnson begin by constructing a commutative Banach algebra - the disentangling algebra - in which rule (R2) above can be applied in a rigorous fashion. An elementary version of the disentangling map is then defined from the commutative setting of the disentangling algebra to the noncommutative Banach algebra of bounded linear operators on a Banach space $X$. This formalism permits the rule (R3) to be carried out in various circumstances. The paper [5] extends the definition of the disentangling map to the time-dependent setting and also to the formation of functions in the presence of the generator of a $\left(C_{0}\right)$ semigroup of linear operators. The advantage of the constructions in 3], [5], and 8] which are outlined below is that the heuristic 'rules' of Feynman stated above can be rigorously applied at each step.

Another paper related to the current article is the paper by DeFacio, Johnson, and Lapidus [1] which addresses the application of Feynman's 'rules' to exponential functions of several noncommuting bounded linear operators as well as evolution equations satisfied by these exponential functions. Also found in [1] is the use of measures to attach time indices to operators (rule (R1)). These measures give us the relative position of the operators in the final disentangled expressions. For example, if we have operators $A_{1}, \ldots, A_{k}$ and associated measures $\mu_{1}, \ldots, \mu_{k}$ for which the support of $\mu_{j}$ lies to the left of the support of $\mu_{j+1}$, then the operator $A_{j}$ always acts prior to $A_{j+1}$. Of course, if the ordering of these supports is changed, then the order of the operators is also changed. Hence an entire family of functional calculi is indexed by the time-ordering measures $\mu_{1}, \ldots, \mu_{k}$. One can find a detailed exposition of this approach to the attachment of time indices in [7] as well in the memoir [6].

As can be seen in [3], [5, 8], and [9], the disentangling map is a continuous contraction from the disentangling algebra to the noncommutative Banach algebra of bounded linear operators on a Banach space. What we propose to do in the present paper is investigate the effects of absolute continuity on the disentangling map. In particular, if we associate continuous Borel probability measures $\mu_{1}, \ldots, \mu_{k}$ on, say, the interval $[0, T]$, to the operators $A_{1}, \ldots, A_{k}$ and if we assume that $\nu_{1}, \ldots, \nu_{k}$ are continuous Borel probability measures on $[0, T]$ for which $\mu_{i} \ll \nu_{i}$, then it is natural to ask what effect this assumption has on the disentangling map. As will be seen below, one effect will be the change in the domain of the disentangling map as we pass from the $k$-tuple of $\mu$ 's to the $k$-tuple of $\nu$ 's. Strictly speaking, the map itself changes albeit in a natural way and in fact this presents no problem. In the simplest case we consider, namely the case where the measures are probability measures, we will see that while the form of the domain changes, the domains can be identified, i.e. they are essentially the same. However we will be unable to identify the domains when the time-ordering measures are not probability measures. What will happen in this setting is that we can apply the disentangling maps corresponding to the two $n$-tuples of measures to a subalgebra of the one of the disentangling algebras. Finally, in the setting where we have time dependent operator-valued functions 
$A_{1}(s), \ldots, A_{k}(s)$, we will be unable to find any reasonable relation between the domains of the disentangling maps corresponding to the two $k$-tuples of measures. Here we will slightly redefine the domain of one of the disentangling maps in order to obtain a theorem which again expresses the same end result as those mentioned above. In each of the settings described above, once we have identified the algebra of functions to which we can apply the disentangling maps corresponding to the two $k$-tuples of measures, the equation relating the action of these maps will have the same form.

\section{NotATiOn AND DEFINITIONS}

In what follows we carefully define the disentangling map. The first definitions construct the domain of the disentangling map as a commutative Banach algebra. This Banach algebra will supply the commutative setting in which Feynman's rules stated in the introduction can be applied in a rigorous fashion.

Definition 2.1. Let $r_{1}, \ldots, r_{k}$ be positive real numbers. Define $\mathbb{A}\left(r_{1}, \ldots, r_{k}\right)$ to be the set of all complex-valued functions of $k$ complex variables which are analytic at $(0, \ldots, 0)$ and are such that their power series expansion

$$
f\left(z_{1}, \ldots, z_{k}\right)=\sum_{n_{1}, \ldots, n_{k}=0}^{\infty} a_{n_{1}, \ldots, n_{k}} z_{1}^{n_{1}} \cdots z_{k}^{n_{k}}
$$

converges absolutely, at least on the closed polydisk $\left|z_{1}\right| \leq r_{1}, \ldots,\left|z_{k}\right| \leq r_{k}$. We define a norm on $\mathbb{A}\left(r_{1}, \ldots, r_{k}\right)$ by

$$
\|f\|_{\mathbb{A}}=\sum_{n_{1}, \ldots, n_{k}=0}^{\infty}\left|a_{n_{1}, \ldots, n_{k}}\right| r_{1}^{n_{1}} \cdots r_{k}^{n_{k}} .
$$

With this norm $\mathbb{A}$ is a commutative Banach algebra. (See 3 for a proof of this fact.)

We now define another, essentially identical, Banach algebra. Let $X$ be a Banach space and let $A_{1}, \ldots, A_{k} \in \mathcal{L}(X)$.

Remark 2.1. We will assume throughout that the Banach space $X$ is separable. While this assumption is not needed in the time independent setting, it is necessary for the time dependent setting.

Definition 2.2. Given the operators $A_{1}, \ldots, A_{k}$ above we keep the norms of these operators as weights and disgard the nature of $A_{1}, \ldots, A_{k}$ as operators and introduce formal commuting objects $A_{1}^{\sim}, \ldots, A_{k}^{\sim}$. We define the disentangling algebra $\mathbb{D}\left(A_{1}, \ldots, A_{k}\right)$ to be the collection of all expressions of the form

$$
\begin{aligned}
& f\left(A_{1}^{\sim}, \ldots, A_{k}^{\sim}\right) \\
& =\sum_{n_{1}, \ldots, n_{k}=0}^{\infty} a_{n_{1}, \ldots, n_{k}}\left(A_{1}^{\sim}\right)^{n_{1}} \cdots\left(A_{k}^{\sim}\right)^{n_{k}}
\end{aligned}
$$

where

$$
\|f\|_{\mathbb{D}}:=\sum_{n_{1}, \ldots, n_{k}=0}^{\infty}\left|a_{n_{1}, \ldots, n_{k}}\right|\left\|A_{1}\right\|^{n_{1}} \cdots\left\|A_{k}\right\|^{n_{k}}<\infty .
$$

The expression defined in (4) is a norm under which $\mathbb{D}$ is a commutative Banach algebra (see [3]). 
Remark 2.2. It is the algebra $\mathbb{D}$ that forms the "commutative world" that will be used to do the calculations required by Feynman's rules.

We are now ready to define the action of the disentangling map as a linear operator from $\mathbb{D}\left(A_{1}, \ldots, A_{k}\right)$ to $\mathcal{L}(X)$. To this end, associate to each $A_{i}$ a continuous Borel probability measure $\mu_{i}$ on the interval $[0, T]$. These measures serve to time order the operators as discussed in the introduction. Let

$$
P^{n_{1}, \ldots, n_{k}}\left(A_{1}^{\sim}, \ldots, A_{k}^{\sim}\right)=\left(A_{1}^{\sim}\right)^{n_{1}} \cdots\left(A_{k}^{\sim}\right)^{n_{k}} .
$$

Definition 2.3. We define the action of the disentangling map on

$$
P^{n_{1}, \ldots, n_{k}}\left(A_{1}^{\sim}, \ldots, A_{k}^{\sim}\right)
$$

by

$$
\begin{aligned}
& \mathcal{T}_{\mu_{1}, \ldots, \mu_{k}} P^{n_{1}, \ldots, n_{k}}\left(A_{1}^{\sim}, \ldots, A_{k}^{\sim}\right) \\
& :=\sum_{\pi \in S_{n}} \int_{\Delta_{n}(\pi)} C_{\pi(n)}\left(s_{\pi(n)}\right) \cdots C_{\pi(1)}\left(s_{\pi(1)}\right) \\
& \cdot\left(\mu_{1}^{n_{1}} \times \cdots \times \mu_{k}^{n_{k}}\right)\left(d s_{1}, \ldots, d s_{n}\right)
\end{aligned}
$$

where $n:=n_{1}+\cdots+n_{k}$,

$$
\Delta_{l}(\pi)=\left\{\left(s_{1}, \ldots, s_{l}\right) \in[0, T]^{l}: 0<s_{\pi(1)}<\cdots<s_{\pi(l)}<T\right\},
$$

and

$$
C_{\pi(i)}\left(s_{\pi(i)}\right)= \begin{cases}A_{1}\left(s_{\pi(i)}\right), & \pi(i) \in\left\{1, \ldots, n_{1}\right\}, \\ A_{2}\left(s_{\pi(i)}\right), & \pi(i) \in\left\{n_{1}, \ldots, n_{1}+n_{2}\right\}, \\ \vdots & \\ A_{k}\left(s_{\pi(i)}\right), & \pi(i) \in\left\{n-n_{k}+1, \ldots, n\right\} .\end{cases}
$$

Finally, for $f$ written as in (3) we define

$$
\begin{aligned}
& \mathcal{T}_{\mu_{1}, \ldots, \mu_{k}} f\left(A_{1}(\cdot)^{\sim}, \ldots, A_{k}(\cdot)^{\sim}\right) \\
& =f_{\mu_{1}, \ldots, \mu_{k}}\left(A_{1}(\cdot), \ldots, A_{k}(\cdot)\right) \\
& =\sum_{n_{1}, \ldots, n_{k}=0}^{\infty} a_{n_{1}, \ldots, n_{k}} \\
& \cdot \mathcal{T}_{\mu_{1}, \ldots, \mu_{k}} P^{n_{1}, \ldots, n_{k}}\left(A_{1}(\cdot)^{\sim}, \ldots, A_{k}(\cdot)^{\sim}\right) .
\end{aligned}
$$

Remark 2.3. It is easy to obtain equation (6) using the assumption that our measures are probability measures as well as Feynman's rules that are given in the introduction. One first writes the monomial $\left(A_{1}^{\sim}\right)^{n_{1}} \cdots\left(A_{k}^{\sim}\right)^{n_{k}}$ using integrals with respect to the probability measures involved. Then the time ordering is carried out using the sum over the permutation group. It is here that the formal objects corresponding to the $C_{i}(s)$ make their appearance. However it seems reasonable to state this as a definition at the outset.

The reader will note that the definitions above were written only in the time independent setting. The natural question at this point is how to define the corresponding Banach algebras in the time-dependent setting. The definition we use below was used in [9] and [5]. We first define the disentangling algebra. 
Definition 2.4. Let, for $i=1, \ldots, k, A_{i}:[0, T] \mapsto \mathcal{L}(X)$ be measurable in the sense that $A_{i}^{-1}(E)$ is a Borel set in $[0, T]$ for every strongly open subset $E$ in $\mathcal{L}(X)$. Let $\mu_{1}, \ldots, \mu_{k}$ be continuous Borel probability measures (the time ordering measures) on $[0, T]$ such that

$$
r_{i}:=\int_{[0, T]}\left\|A_{i}(s)\right\|\left|\mu_{i}\right|(d s)<\infty
$$

for $i=1, \ldots, k$. We use these real numbers as weights to form the algebra $\mathbb{A}_{T}\left(r_{1}, \ldots, r_{k}\right)$ as above. We can form the corresponding disentangling algebra by using formal commuting objects $\left(A_{i}(\cdot), \mu_{i}\right)^{\sim}, i=1, \ldots, k$. The disentangling algebra is defined as before using these formal objects.

For a function $f$ written as a power series in these commuting objects, we define the disentangling map exactly as above, that is,

$$
\begin{aligned}
& \mathcal{T}_{\mu_{1}, \ldots, \mu_{k}} f\left(A_{1}(\cdot)^{\sim}, \ldots, A_{k}(\cdot)^{\sim}\right) \\
& =f_{\mu_{1}, \ldots, \mu_{k}}\left(A_{1}(\cdot), \ldots, A_{k}(\cdot)\right) \\
& =\sum_{n_{1}, \ldots, n_{k}=0}^{\infty} a_{n_{1}, \ldots, n_{k}} \\
& \cdot \mathcal{T}_{\mu_{1}, \ldots, \mu_{k}} P^{n_{1}, \ldots, n_{k}}\left(A_{1}(\cdot)^{\sim}, \ldots, A_{k}(\cdot)^{\sim}\right) .
\end{aligned}
$$

Even though the formal objects defined here clearly involve the measures used, we will, for simplicity, continue using the same notation for the formal object in our calculations. It is shown in $[5$ that the definition of the disentangling map given in equation (11) defines a continuous linear contraction from the disentangling algebra $\mathbb{D}_{T}\left(\left(A_{1}(\cdot), \mu_{1}\right)^{\sim}, \ldots,\left(A_{k}(\cdot), \mu_{k}\right)^{\sim}\right)$ to $\mathcal{L}(X)$. In fact, in the time independent setting the disentangling map is a norm one contraction. However, in the time dependent setting it is no longer the case that the disentangling map is of norm one (see [9]).

Remark 2.4. We use a subscript $T$ above in the notation denoting our Banach algebras to stress that we are working on a fixed interval $[0, T]$.

\section{EfFECts of ABSOlute CONTINUity}

We will first address the time independent setting. To this end, let $A_{1}, \ldots, A_{k} \in$ $\mathcal{L}(X)$ and let $\mu_{1}, \ldots, \mu_{k}, \nu_{1}, \ldots, \nu_{k}$ be continuous Borel probability measures on $[0, T]$ for fixed $T$. Associate the measures $\mu_{i}, \nu_{i}$ with $A_{i}$ for $i=1, \ldots, k$. Assume that $\mu_{i} \ll \nu_{i}$ for each $i=1, \ldots, k$.

Theorem 3.1. Let $m_{1}, \ldots, m_{k}$ be nonnegative integers. We have

$$
P_{\mu_{1}, \ldots, \mu_{k}}^{m_{1}, \ldots, m_{k}}\left(A_{1}, \ldots, A_{k}\right)=P_{\nu_{1}, \ldots, \nu_{k}}^{m_{1}, \ldots, m_{k}}\left(\left[\frac{d \mu_{1}}{d \nu_{1}} A_{1}\right], \ldots,\left[\frac{d \mu_{k}}{d \nu_{k}} A_{k}\right]\right) .
$$

Proof. Write $A_{i}(s):=A_{i}$ for all $s \in[0, T]$. Also write

$$
g_{i}:=\frac{d \mu_{i}}{d \nu_{i}}
$$


for each $i=1, \ldots, k$. Note that

$$
\begin{aligned}
\int_{[0, T]}\left\|A_{i}(s)\right\| \mu_{i}(d s) & =\left\|A_{i}(s)\right\| \int_{[0, T]} g_{i}(s) \nu_{i}(d s) \\
& =\left\|g_{i}\right\|_{L^{1}\left([0, T], \nu_{i}\right)}\left\|A_{i}(s)\right\| \\
& =\left\|A_{i}(s)\right\| .
\end{aligned}
$$

This calculation shows that

$$
\mathbb{A}_{T}\left(\left\|A_{1}\right\|, \ldots,\left\|A_{k}\right\|\right)=\mathbb{A}_{T}\left(R_{1}, \ldots, R_{k}\right)
$$

where

$$
R_{i}:=\int_{[0, T]}\left\|g_{i}(s) A_{i}\right\| \nu_{i}(d s)
$$

for $i=1, \ldots, k$. In fact, equation (16) shows us that the weights $R_{i}$ are just the $L^{1}\left(\nu_{i}\right)$ norms of the operator-valued functions $g_{i}(s) A_{i}$ and referring to Definition 2.4 it becomes clear that the weights $R_{i}$ are the weights for the algebra $\mathbb{A}_{T}$ with the operator-valued functions $g_{i}(s) A_{i}$.

Now that we have equation (15) we need to determine how this equation relates the disentangling algebras $\mathbb{D}_{T}\left(A_{1}^{\sim}, \ldots, A_{k}^{\sim}\right)$ and $\mathbb{D}_{T}\left(\left(g_{1} \cdot A_{1}\right)^{\sim}, \ldots,\left(g_{k} \cdot A_{k}\right)^{\sim}\right)$. From [3] we know that the algebras $\mathbb{A}_{T}\left(\left\|A_{1}\right\|, \ldots,\left\|A_{k}\right\|\right)$ and $\mathbb{D}_{T}\left(A_{1}^{\sim}, \ldots, A_{k}^{\sim}\right)$ can be identified via the map

$$
f\left(z_{1}, \ldots, z_{k}\right) \mapsto f\left(A_{1}^{\sim}, \ldots, A_{k}^{\sim}\right) .
$$

It is clear that the algebras $\mathbb{A}_{T}\left(R_{1}, \ldots, R_{k}\right)$ and $\mathbb{D}_{T}\left(\left(g_{1} \cdot A_{1}\right)^{\sim}, \ldots,\left(g_{k} \cdot A_{k}\right)^{\sim}\right)$ can be identified by essentially the same map, i.e.

$$
f\left(z_{1}, \ldots, z_{k}\right) \mapsto f\left(\left(g_{1} \cdot A_{1}\right)^{\sim}, \ldots,\left(g_{k} \cdot A_{k}\right)^{\sim}\right) .
$$

Hence it is clear that our two disentangling algebras can be identified and we will treat the identification as an equality.

Now that we know that the disentangling maps being applied on the right and left sides of equation (12) have the same domain, we will proceed to establish the equality of these two expressions. To this end, let $m_{1}, \ldots, m_{k}$ be nonnegative integers. We have, using our absolute continuity assumption,

$$
\begin{gathered}
\left(A_{1}^{\sim}\right)^{m_{1}} \cdots\left(A_{k}^{\sim}\right)^{m_{k}}= \\
=\left\{\int_{[0, T]} A_{1}^{\sim}(s) \mu_{1}(d s)\right\}^{m_{1}} \cdots\left\{\int_{[0, T]} A_{k}^{\sim}(s) \mu_{k}(d s)\right\}^{m_{k}} \\
\left.\cdots\left\{\int_{[0, T]} A_{1}^{\sim}(s) g_{1}(s) \nu_{1}(d s)\right\}^{m_{1}}(s) \nu_{k}(d s)\right\}^{m_{k}} \\
=\left\{\int_{[0, T]}\left(g_{1} \cdot A_{1}\right)^{\sim}(s) \nu_{1}(d s)\right\}^{m_{1}} \\
\cdots\left\{\int_{[0, T]}\left(g_{k} \cdot A_{k}\right)^{\sim}(s) \nu_{k}(d s)\right\}^{m_{k}} .
\end{gathered}
$$


It is now clear that the disentangled expressions involving the formal objects satisfy

$$
\left[P^{m_{1}, \ldots, m_{k}}\left(A_{1}^{\sim}, \ldots, A_{k}^{\sim}\right)\right]_{\mu_{1}, \ldots, \mu_{k}}=\left[P^{m_{1}, \ldots, m_{k}}\left(\left(g_{1} \cdot A_{1}\right)^{\sim}, \ldots,\left(g_{k} \cdot A_{k}\right)^{\sim}\right)\right]_{\nu_{1}, \ldots, \nu_{k}}
$$

where the left side of (20) is calculated using the measures $\mu_{1}, \ldots, \mu_{k}$ and the right side is calculated using the measures $\nu_{1}, \ldots, \nu_{k}$. Moreover, with

$$
\left[\tilde{C}_{i}(s)\right]_{\mu_{1}, \ldots, \mu_{k}}= \begin{cases}A_{1}^{\sim}(s) & \text { if } i \in\left\{1, \ldots, m_{1}\right\}, \\ A_{2}^{\sim}(s) & \text { if } i \in\left\{m_{1}+1, \ldots, m_{1}+m_{2}\right\}, \\ \vdots & \\ A_{\tilde{k}}^{\sim}(s) & \text { if } i \in\left\{m_{1}+\cdots+m_{k-1}+1, \ldots, m\right\},\end{cases}
$$

and

$$
\left[\tilde{C}_{i}(s)\right]_{\nu_{1}, \ldots, \nu_{k}}= \begin{cases}\left(g_{1} \cdot A_{1}\right)^{\sim}(s) & \text { if } i \in\left\{1, \ldots, m_{1}\right\}, \\ \left(g_{2} \cdot A_{2}\right)^{\sim}(s) & \text { if } i \in\left\{m_{1}+1, \ldots, m_{1}+m_{2}\right\}, \\ \vdots & \\ \left(g_{k} \cdot A_{k}\right)^{\sim}(s) & \text { if } i \in\left\{m_{1}+\cdots+m_{k-1}+1, \ldots, m\right\},\end{cases}
$$

and the "usual" definitions of $\left[C_{i}(s)\right]_{\mu_{1}, \ldots, \mu_{k}}$ and $\left[C_{i}(s)\right]_{\nu_{1}, \ldots, \nu_{k}}$ (i.e. obtain the appropriate operator by erasing the tilde) we see that the asserted equality holds.

The previous theorem can be immediately generalized to the following.

Theorem 3.2. Under all of the hypotheses of Theorem 3.1 the maps $\mathcal{T}_{\mu_{1}, \ldots, \mu_{k}}$ : $\mathbb{D}_{T}\left(A_{1}^{\sim}, \ldots, A_{k}^{\sim}\right) \mapsto \mathcal{L}(X)$ and $\mathcal{T}_{\nu_{1}, \ldots, \nu_{k}}: \mathbb{D}_{T}\left(\left(g_{1} \cdot A_{1}\right)^{\sim}, \ldots,\left(g_{k} \cdot A_{k}\right)^{\sim}\right) \mapsto \mathcal{L}(X)$ satisfy

$$
\mathcal{T}_{\mu_{1}, \ldots, \mu_{k}} f\left(A_{1}^{\sim}, \ldots, A_{k}^{\sim}\right)=\mathcal{T}_{\nu_{1}, \ldots, \nu_{k}} f\left(\left(g_{1} \cdot A_{1}\right)^{\sim}, \ldots,\left(g_{k} \cdot A_{k}\right)^{\sim}\right) .
$$

Proof. The proof is accomplished by applying Theorem 3.1 to each term of the power series expansion of the function $f$.

Corollary 3.1. Assume the hypotheses of the previous theorem. Suppose that $\nu_{1}=$ $\cdots=\nu_{k}=\nu$, i.e. $\mu_{i} \ll \nu$ for each $i$. Then

$$
\mathcal{T}_{\mu_{1}, \ldots, \mu_{k}} f\left(A_{1}^{\sim}, \ldots, A_{k}^{\sim}\right)=\mathcal{T}_{\nu, \ldots, \nu} f\left(\left(g_{1} \cdot A_{1}\right)^{\sim}, \ldots,\left(g_{k} \cdot A_{k}\right)^{\sim}\right)
$$

where now $g_{i}=\frac{d \mu_{i}}{d \nu}$ for each $i$.

Theorem [3.2, in particular equation (23), establishes the relationship between two $k$-tuples of measures where each measure in one $k$-tuple is absolutely continuous with respect to the corresponding measure in the other $k$-tuple. As might be expected, this relation continues to hold even when we move to the setting where we no longer have probability measures serving as time-ordering measures. The change will be in the domains of the disentangling maps used on each side of (23).

Remark 3.1. In what follows we use non-probability measures to time-order the operators. As is shown in [3], the use of non-probability measures does not change the disentangled expressions. However, the weights used do change somewhat and we will follow the notation used in [3] for the disentangling algebras that result. (In 
fact, the easiest way to see how the weights change is to look at equation (10) and assume that the operator-valued function is constant. The weights are then of the form $\left\|A_{i}\right\|\left|\mu_{i}\right|([0, T])$.) More specifically, we apply this notation below in equation (27). This notation also stresses the fact that the measures are not probability measures.

Let $A_{1}, \ldots, A_{k} \in \mathcal{L}(X)$ and let $\mu_{1}, \ldots, \mu_{k}$ be continuous Borel measures on $[0, \infty)$ which are such that $\left|\mu_{i}\right|([0, T])<\infty$ for every $T>0$ and recall that the weights $r_{1}, \ldots, r_{k}$ for the commutative Banach algebra $\mathbb{A}_{T}\left(r_{1}, \ldots, r_{k}\right)$ are given in equation (10). (Even though these weights are defined for the time-dependent disentangling map, it is convenient to consider our operators here as constant operatorvalued functions on $[0, T]$ and use these weights in our discussion.) Using equation (10) we have

$$
r_{j}=\left|\mu_{j}\right|([0, T])\left\|A_{j}\right\| .
$$

It is shown in [4] that even when non-probability measures are used, the application of Feynman's rules gives us identical results. Consequently the disentangling map is defined exactly as in the probability measure case. The difference is in the domain of the disentangling map, and this is illustrated by equation (10) where the effect of the non-probability measure is clearly seen. Now let $\nu_{1} \ldots, \nu_{k}$ be continuous Borel measures on $[0, \infty)$ satisfying the same conditions as imposed on the measures $\mu_{i}$. Assume that, for each $i=1, \ldots, k, \mu_{i} \ll \nu_{i}$. Then a simple calculation shows that, for each $i$,

$$
\begin{aligned}
\left|\mu_{i}\right|([0, T])\left\|A_{i}\right\|= & \left\|A_{i}\right\| \int_{[0, T]}\left|\mu_{i}\right|(d s) \\
& =\left\|A_{i}\right\| \int_{[0, T]}\left|\frac{d \mu_{i}}{d \nu_{i}}\right|\left|\nu_{i}\right|(d s) \\
& \leq\left\|g_{i} \cdot A_{i}\right\|_{L^{\infty}\left([0, T], \mathcal{L}(X),\left|\nu_{i}\right|\right)}\left\|\nu_{i}\right\| \\
& =:\left\|g_{i} \cdot A_{i}\right\|_{\infty}\left\|\nu_{i}\right\|
\end{aligned}
$$

where we use the notation $\|\mu\|$ for $|\mu|([0, T])$. From this calculation it is clear that $\mathbb{A}_{T}\left(r_{1}, \ldots, r_{k}\right) \supseteq \mathbb{A}_{T}\left(\left\|g_{1} \cdot A_{1}\right\|_{\infty}, \ldots,\left\|g_{k} \cdot A_{k}\right\|_{\infty}\right)$. This inclusion clearly induces an embedding

$$
\mathbb{D}_{T}\left(\left\|\nu_{1}\right\|\left(g_{1} \cdot A_{1}\right)^{\sim}, \ldots,\left\|\nu_{k}\right\|\left(g_{k} \cdot A_{k}\right)^{\sim}\right) \hookrightarrow \mathbb{D}_{T}\left(\left\|\mu_{1}\right\| A_{1}^{\sim}, \ldots,\left\|\mu_{k}\right\| A_{k}^{\sim}\right) .
$$

Via this embedding we can consider the first disentangling algebra in (27) to be a subalgebra of the second. We can now state our next theorem.

Theorem 3.3. Let $\mathcal{T}_{\mu_{1}, \ldots, \mu_{k}}: \mathbb{D}_{T}\left(\left\|\mu_{1}\right\| A_{1}^{\sim}, \ldots,\left\|\mu_{k}\right\| A_{k}^{\tilde{k}}\right) \rightarrow \mathcal{L}(X)$. For $f \in$ $\mathbb{D}_{T}\left(\left\|\nu_{1}\right\|\left(g_{1} \cdot A_{1}\right)^{\sim}, \ldots,\left\|\nu_{k}\right\|\left(g_{k} \cdot A_{k}\right)^{\sim}\right)$, we have

$$
\mathcal{T}_{\mu_{1}, \ldots, \mu_{k}} f\left(A_{1}^{\sim}, \ldots, A_{k}^{\sim}\right)=\mathcal{T}_{\nu_{1}, \ldots, \nu_{k}} f\left(\left(g_{1} \cdot A_{1}\right)^{\sim}, \ldots,\left(g_{k} \cdot A_{k}\right)^{\sim}\right) .
$$

Proof. The proof is almost immediate. The disentangling map as defined when nonprobability measures are present gives identical results as obtained using probability measures. Hence the proof is clear.

\section{Effects of Absolute Continuity in the time-Dependent SETting}

The reader will recall that the disentangling map $\mathcal{T}_{\mu_{1}, \ldots, \mu_{k}}$ was defined above in both the time-independent setting as well as in the time-dependent setting. 
Moreover, after reading the previous section, it is natural to inquire as to whether or not the same type of statements can be obtained in the presence of operator-valued functions, i.e. the time-dependent case. The answer is yes, one can indeed write an equation which is exactly the same as equation (28). However, the theorem we will record is not as satisfying in that there is no natural relation to be found between the domains of the disentangling maps involved. What we will do is redefine the weights we use in the construction of the commutative Banach algebras of functions which give us the disentangling algebras.

Let $A_{i}:[0, T] \rightarrow \mathcal{L}(X), i=1, \ldots, k$, be measurable in the sense of Definition 2.4. Recall that we are assuming that the Banach space $X$ is separable. Associate with $A_{i}$ the measures $\mu_{i}, \nu_{i}, i=1, \ldots, k$, which are continuous Borel probability measures on $[0, T]$. Assume that, for each $i=1, \ldots, k, \mu_{i} \ll \nu_{i}$. For the measures $\mu_{1}, \ldots, \mu_{k}$ denote by $r_{1}^{\mu}, \ldots, r_{k}^{\mu}$ the weights for the algebra $\mathbb{A}_{T}\left(r_{1}^{\mu}, \ldots, r_{k}^{\mu}\right)$. We will use $r_{1}^{\nu}, \ldots, r_{k}^{\nu}$ for the weights corresponding to the measures $\nu_{i}$. Recall that, for each $i$,

$$
r_{i}^{\mu}=\int_{[0, T]}\left\|A_{i}(s)\right\|\left|\mu_{i}\right|(d s)
$$

We define

$$
r_{i}^{\nu}:=\int_{[0, T]}\left\|A_{i}(s)\right\|\left|\frac{d \mu_{i}}{d \nu_{i}}\right|\left|\nu_{i}\right|(d s)=\int_{[0, T]}\left\|\frac{d \mu_{i}}{d \nu_{i}} \cdot A_{i}(s)\right\|\left|\nu_{i}\right|(d s)
$$

for each $i$ where the absolute continuity assumption has been used in the second equality. Note that the number we are using for $r_{i}^{\nu}$ is different from the definition of weights given in the introduction and recalled above in equation (29). If we had used the original definition, we would have

$$
r_{i}^{\nu}=\int_{[0, T]}\left\|A_{i}(s)\right\|\left|\nu_{i}\right|(d s) .
$$

However, with this definition of the weights $r_{i}^{\nu}$, we cannot obtain a convenient relationship between the two sets of weights. In fact, all that can be said is that

$$
r_{i}^{\mu} \leq\left\|\frac{d \mu_{i}}{d \nu_{i}}\right\|_{\infty} r_{i}^{\nu}
$$

which only makes sense if we assume that the essential supremum of the derivative above is bounded. Further, this inequality does not enable us to obtain any kind of natural containment of disentangling algebras to be obtained. Consequently, we will use the weights given in equations (29) and (30) to determine the disentangling algebras. It is clear that the absolute continuity assumption guarantees that $r_{i}^{\mu}=r_{i}^{\nu}$ for each $i$. As in the proof of Theorem 3.1 this enables us to identify the disentangling algebras $\mathbb{D}_{T}\left(\left(A_{1}(\cdot), \mu_{1}\right)^{\sim}, \ldots,\left(A_{k}(\cdot), \mu_{k}\right)^{\sim}\right)$ and $\mathbb{D}_{T}\left(\left(\frac{d \mu_{1}}{d \nu_{1}} \cdot A_{1}(\cdot), \nu_{1}\right)^{\sim}, \ldots,\left(\frac{d \mu_{k}}{d \nu_{k}} \cdot A_{k}(\cdot), \nu_{k}\right)^{\sim}\right)$.

With these preliminaries out of the way, we can state the following.

Theorem 4.1. Let $A_{i}(\cdot), \mu_{i}$, and $\nu_{i}, i=1, \ldots, k$, be as above. Define weights $r_{i}^{\mu}, r_{i}^{\nu}$ by equations (29) and (30). Then the disentangling algebra

$$
\mathbb{D}_{T}\left(\left(A_{1}(\cdot), \mu_{1}\right)^{\sim}, \ldots,\left(A_{k}(\cdot), \mu_{k}\right)^{\sim}\right)
$$


can be identified with the disentangling algebra

$$
\mathbb{D}_{T}\left(\left(\frac{d \mu_{1}}{d \nu_{1}} \cdot A_{1}(\cdot), \nu_{1}\right)^{\sim}, \ldots,\left(\frac{d \mu_{k}}{d \nu_{k}} \cdot A_{k}(\cdot), \nu_{k}\right)^{\sim}\right) .
$$

Moreover, for any $f$ in these (essentially identical) disentangling algebras we have

$$
\mathcal{T}_{\mu_{1}, \ldots, \mu_{k}} f\left(A_{1}^{\sim}(\cdot), \ldots, A_{k}^{\sim}(\cdot)\right)=\mathcal{T}_{\nu_{1}, \ldots, \nu_{k}} f\left(\left[\frac{d \mu_{1}}{d \nu_{1}} \cdot A_{1}(\cdot)\right]^{\sim}, \ldots,\left[\frac{d \mu_{k}}{d \nu_{k}} \cdot A_{k}(\cdot)\right]^{\sim}\right) .
$$

Proof. As before, once the domains of the disentangling maps have been identified, the proof is a straightforward calculation.

\section{FinAl REMARKS}

At this point it is worth briefly commenting upon the utility of the results presented above. The author's present work has been primarily concerned with establishing stability properties for Feynman's operational calculus. (See [8], [9], [10].) In particular, the study of stability with respect to the time-ordering measures $\mu_{1}, \ldots, \mu_{k}$ and with respect to the operators $A_{1}, \ldots, A_{k}$ made it clear that stability with respect to the operators was generally easier to establish. With this in mind, the author was naturally led to consider the topics above to enable the transfer of the study of the convergence of sequences of measures to the study of the convergence of the corresponding sequences of operator-valued functions (via Theorem 3.2). Work along these lines is in progress. The results presented above also show much promise in simplifying disentangling calculations in certain special cases. For instance, when all of the time-ordering measures are absolutely continuous with respect to one measure (Lebesgue measures for example), the integrals in the disentangling can be expressed as integrals with respect to one measure as opposed to a $k$-tuple of measures.

\section{REFERENCES}

1. Defacio, B., Johnson, G.W., and Lapidus, M.L., Feynman's operational calculus and evolution equations. Acta Applicandae Mathematicae 47 (1997), 155 - 211. MR 98f:58050

2. Feynman, R.P., An operator calculus having applications in quantum electrodynamics, Phys. Rev. 84 (1951), 108-128. MR 13:410e

3. Jefferies, B., and Johnson, G.W., Feynman's operational calculi for noncommuting operators: definitions and elementary properties, Russ. J. Math. Phys. 8 (2001), to appear.

4. - Feynman's operational calcui for noncommuting operators: tensors, ordered support and disentangling an exponential factor, Math. Notes 70 (2001), accepted for publication.

5. Jefferies, B., Johnson, G.W., and Nielsen, L., Feynman's operational calculi for timedependent noncommuting operators. J. of Korean Math. Soc. 38 (2001), 193-226. MR 2001m:81154

6. Johnson, G.W., and Lapidus, M.L., Generalized Dyson series, generalized Feynman diagrams, the Feynman integral and Feynman's operational calculus, Mem. Amer. Math. Soc. 62 (1986), 1-78. MR 88f:81034

7. — The Feynman Integral and Feynman's Operational Calculus. Oxford Science Publications, Oxford Mathematical Monographs, Oxford Univ. Press, Oxford and New York, 2000. MR 2001i: 58015

8. Johnson, G.W., Nielsen, L., A stability theorem for Feynman's operational calculus, Conference Proc. Canadian Math. Soc.: Conference in honor of Sergio Albeverio's 60th birthday, 2000. MR 2001k: 47020 
9. Nielsen, L., Stability Properties for Feynman's Operational Calculus, Ph.D. Dissertation, Mathematics, University of Nebraska, Lincoln, 1999.

10. Nielsen, L., Stability properties of Feynman's operational calculus for exponential functions of noncommuting operators, submitted for publication.

Department of Mathematics and Computer Science, Creighton University, Omaha, NEBRASKA 68178-2090

E-mail address: lnielsen@creighton.edu 